\section{Factor people into tiger conservation}

Tiger populations in India and Nepal have increased by almost one-third and two-thirds, respectively, over the past 5 years. But for these countries to achieve their goal of doubling their combined tiger numbers to more than 3,600 by 2022 , they must take the human population into account as well.

India is projected to become the world's most populous nation by 2028 , with around 1.45 billion people. This will place further pressure on tiger habitats such as those in Arunachal Pradesh and Uttarakhand, where hydropower projects are planned.

Local village populations are unlikely to cooperate with tiger conservation efforts while the animals continue to take livestock and kill humans (N. H. Carter et al. Ambio 43, 125-137; 2014). In India alone, tigers killed 822 people between 1990 and 2009 (N.P.S. Chauhan Julius Kühn Arch. http://doi. org/46k; 2011).

It is crucial for the Indian and Nepalese governments to install protection for villagers against tigers and to provide them with incentives to participate in tiger conservation. Improved management of existing reserves and more conservation areas are also a priority.

Achyut Aryal ${ }^{\star}$ Massey

University, Auckland,

New Zealand.

a.aryal@massey.ac.nz

${ }^{*}$ On behalf of 5 correspondents (see go.nature.com/cr4jli for full list).

\section{Healthy diet sustains the environment too}

The idea that dietary recommendations for humans are compatible with an environmentally sustainable food system is supported by a 2015 report from the US National Academy of Sciences (go.nature.com/t2ovdt).

The implication that dietary recommendations should take the environmental impact of food production into account caused controversy earlier this year. Parts of the US government and the food industry accused the federal Dietary Guidelines Advisory Committee of going beyond its remit in advocating a more plantbased diet that would be better for sustainability as well as human health (see go.nature.com/lcwj6i).

Scientists, policy-makers and other stakeholders must move beyond considering health, environmental, social and economic effects in isolation. In recognizing the need to address the food system's complexities and its competing demands and trade-offs, the National Academy of Sciences' report has already been the subject of briefings to Congress and US governmental agencies.

Malden Nesheim Cornell

University, Ithaca, New York, USA.

Patrick J. Stover Cornell

University, Ithaca, New York; and American Society for Nutrition, Bethesda, Maryland, USA.

Maria Oria The National Academies, Washington DC, USA. pjs13@cornell.edu

\section{Beware climate neo-scepticism}

We coin 'neo-scepticism' as a term to describe an emerging science-policy argument that opposes major efforts to mitigate climate change. Neo-sceptics do not deny anthropogenic global warming, but minimize its projected effects and see mitigation efforts as unjustifiable (see, for example, S. E. Koonin Wall Street Journal 19 September 2014; J. Curry Wall Street Journal 9 October 2014).

Neo-sceptic arguments emphasize well-known uncertainties in climate science, particularly over predicted temperature changes. This diverts attention from the risks of events with extreme biological and economic consequences and from the risks of 'tipping points' with large, nonlinear responses.

Neo-sceptics recognize the importance of policy decisions - who should do what, when, and at what cost, for example. However, they project an ironic certainty in advising the postponement of serious mitigation efforts until confidence intervals for climate projections are narrowed.

Physicists, biologists and social scientists should be focusing more on risks, rather than simply pursuing more-precise estimates of physical parameters that are not linearly related to the most worrisome hazards. Society does not demand certainty to cope with other high-consequence risks, such as those relating to public health, airline safety, terrorism, and natural or technological disasters.

John H. Perkins* The Evergreen State College, Kensington, California, USA.

perkinsj@evergreen.edu

${ }^{*}$ On behalf of 4 correspondents (see go.nature.com/mg7ugffor full list).

\section{Chinese scientists are sharing data}

We cannot agree with Zheng Wan's view that it is getting harder for scientists in China to access high-quality public data (Nature 520, 587; 2015). There are still technical issues to be resolved, but the trend is towards a greater amount of open data - not less.

The Chinese government has established several national data infrastructures over the past few years that are publicly accessible. These include the National Science and Technology Infrastructures organization (www.escience.gov.cn), which has been sharing data since 2009 in fields such as Earth systems, marine science, meteorology, agriculture, forestry, medicine and health. One of its 23 projects is the National Specimen Information Infrastructure (www.nsii.org.cn), launched in 2013, which currently archives data from more than 10.5 million biological, mineral, rock and fossil specimens.

The degree and success of data sharing also rely on individual scientists' attitudes and practices. Many researchers - including some who are publicly funded in China - unfortunately often resist sharing data, actively or passively. They should instead take more responsibility for cultivating a sustainable, 'bottom-up' culture of sharing. Xiaolei Huang Fujian Agriculture and Forestry University, Fuzhou, China. Keping Ma Institute of Botany, Chinese Academy of Sciences, Beijing, China. huangxl@fafu.edu.cn

\section{Star academics in favour of divestment}

Your portrayal of the campus carbon-divestment movement could have provided a more balanced account of the controversy (Nature 521, 16-17; 2015). The strongest resistance to this movement is found not among faculty members, but in the boardrooms of the managing corporations.

As for the former, our own group, Harvard Faculty for Divestment (harvardfacultydivest.com), has grown in less than a year to more than 250 names, and includes several renowned climate scientists and Nobel laureates, as well as members of every school in the Harvard community. James Recht Harvard Medical School, Boston, Massachusetts, USA.

james_recht@hms.harvard.edu

\section{CORRECTION}

In the Outlook article 'Wild bees: Lone rangers' (Nature 521, S62-S63; 2015), entomologist Jordi Bosch was incorrectly affiliated with the University of Barcelona; his affiliation should have read the Autonomous University of Barcelona. 